\title{
Behavioural Consequences of Frontal Cortex Grafts and Enriched Environments after Sensorimotor Cortex Lesions
}

\author{
Michael A. Christie and John C. Dalrymple-Alford \\ Department of Psychology, University of Canterbury, Christchurch 1, New Zealand
}

\section{SUMMARY}

Past studies have experienced difficulty in achieving graft survival and behavioural recovery after sensorimotor cortex lesions. In the present work, adult female rats trained preoperatively to cross a narrow beam for food reward were maintained in standard group cages or an enriched environment, commencing one week after a unilateral lesion. One month post-lesion, half of these rats received multiple suspension grafts of (E20) fetal frontal cortex, placed adjacent to the lesion cavity, and 8 days later recovery of beam-walking skills was examined for a six-week period. The grafts survived in all cases with an appropriate lesion, a notable result given the one month lesion-graft delay, but graft volume was not influenced by postoperative environment. The substantial lesion-induced deficits evident just prior to differential housing showed a marked reduction by the start of post-graft testing, but relative to intact controls a persistent deficit in foot slip errors occurred in all lesion groups. Irrespective of graft status, postoperative enrichment prevented the occurrence of severe foot slips, especially early in retraining. The frontal grafts, however, enhanced beam-walking recovery by reducing the overall frequency of foot slips on early post-grafting sessions, an effect we suggest is related to graft-derived trophic influences, but this measure was not significantly improved by postoperative enrichment.

\section{Reprint address:}

John C. Dalrymple-Alford

Department of Psychology

University of Canterbury

Christchurch 1, New Zealand

\section{KEY WORDS}

sensorimotor cortex, lesions, frontal grafts, enriched environments, recovery of function, behaviour

\section{INTRODUCTION}

Substantial published work has shown that neural grafts promote recovery of behavioural function in a variety of lesion models $/ 6,15 /$. Attaining graft survival and especially recovery of function after cortex lesions has been relatively problematic, however, particularly after sensorimotor cortex (SMC) damage /20/. Whereas cortex transplants show good survival, integration and host-graft reciprocal connections when grafted in neonatal rats with SMC lesions /2-4,23,26,35/, grafts placed in adult rats show either poor survival /31/ or fewer reciprocal connections /10,11,30/. In terms of behaviour, there is relatively little evidence of graft-mediated improvements after SMC lesions. One study achieved improvements in forelimb dexterity after grafts made in neonate rats $/ 26 /$, while a second neonate study, which had poor graft survival (33\%) and no lesion-only group, relied on evidence that successful graft removal produced a lever-pressing deficit reminiscent of a SMC lesion effect in 3 out of 6 rats $/ 27 /$. The standard measure of the effects of large SMC lesions is a deficit on a constrained locomotor task, such as crossing a narrow beam, but previous work with both neonate and adult rats has found no amelioration of beam walking impairments by cortical grafts except in one instance when the adult rats received additional GM1 ganglioside treatment $/ 31,35 /$.

In the case of SMC damage, then, it may be necessary to use additional treatments both to maximise the survival of the grafts and to enhance 
their potential to promote behavioural recovery, especially in adult recipients. One approach that may be valuable in this context is to place the subjects in an enriched postoperative environment. It is known that environmental factors such as enriched housing conditions may produce sparing, recovery and/or compensation of behavioural deficits after brain injury in young or adult rats, particularly after cortical damage $15,36 /$. It is especially pertinent that postoperatively enriched rats with SMC lesions show a markedly less severe deficit in run times on a beam task than do rats housed in more restricted conditions $/ 14 /$, so conjunctive treatment with neural grafting and postoperative enrichment may be particularly useful to promote recovery after SMC damage.

The idea that postoperative enrichment may enhance the functional capabilities of neural transplants in adult rats is supported by work with basal forebrain lesion models. In an anatomical study, enriched housing by comparison to standard conditions produced an early enhancement of fibre outgrowth of cholinergic tissue grafted to the frontal neocortex $/ 9 /$ In behavioural work, cholinergic grafts attenuated maze-learning deficits after fimbria-fornix lesions only in rats that were housed in an enriched postoperative environment, not in those maintained in standard housing conditions $/ 16 /$.

In the present study, we examined the effects of neural grafts in conjunction with an enriched or standard postoperative environment on recovery of beam-walking performance in adult rats given unilateral SMC lesions, using multiple suspension grafts of fetal frontal cortex in the dorsomedial parenchyma ipsilateral to the lesion. Frontal cortex implants can enhance recovery when placed in an ectopic site, even when the appropriate homologous tissue fails to do so $/ 20 /$, and the only evidence thus far for any recovery on a beam task after SMC lesions has been with fetal frontal tissue rather than presumptive SMC, albeit only when ganglioside treatment was used after grafting /31/.

We considered that graft survival may be enhanced by the use of parenchymal suspension grafts because they integrate more readily into the host environment than do solid grafts /1/. Poor graft survival in previous work may have been due to the use of solid cortical grafts placed in the lesion cavity
/31/; better survival is reported with solid tissue placed in the intact adult SMC /28,21/. Even solid cortical grafts develop few characteristics of the adult organotypic organization $/ 11,20,21,33 /$, so there is no particular concern that normal development of cortical tissue may be unduly restricted by using suspension grafts.

For neural transplants to be an effective therapy for brain injury it is likely that they will need to be introduced only after a more substantial post-lesion delay than is common in experimental neural transplant work. In the clinical arena, time is needed for an initial assessment, for the secondary consequences of trauma to diminish and any early "spontaneous recovery" to occur, and it is in this period when an environmental therapy may be more appropriate $/ 34 /$. In the present study, we introduced rats to different environments commencing 7 days after lesion surgery and used a 4-week lesion-graft delay, despite some evidence that survival of frontal cortex grafts may be poor after a prolonged delay, at least with solid implants placed in a frontal lesion cavity $/ 34 /$. It is possible that postoperative enrichment may improve the survival of cortical grafts and that the influence of a long post-lesion delay may be less of an issue with suspension grafts. Work with intrahippocampal septal grafts shows that in some instances adequate survival can be obtained even after lengthy postlesion delays $/ 7 /$.

An additional benefit of a 4-week delay is to focus on the possible effects of graft-derived neurotrophic influences in a situation when the contributions of host neurotrophic factors are minimal. Although brain injury in adult rats induces the release of endogenous trophic factors which may peak 7-10 days after injury and enhance graft survival in some situations $/ 24,55 /$, fetal tissues, particularly frontal cortex, also liberate trophic factors that may exert some influence on host brain and behavioural recovery $/ 8,19,20,34$ /

\section{MATERIALS AND METHODS}

\section{Animals and environments}

Forty-six adult female Sprague-Dawley rats, 100 days old at the start of the experiment, were placed 
on a restricted food schedule to maintain their body weight at $85 \%$, except for 3 days of increased food access to facilitate post-lesion recovery. Water was available ad libitum at all times. Rats were kept in a temperature-controlled colony room under a reversed light schedule and housed in groups of three in standard plastic cages $(51 \times 33.5 \times 18.5$ $\mathrm{cm}$ ), except for those housed in enriched environments for the remainder of the study after lesion surgery.

The rats were placed in either an enriched environment cage or in new groups in a fresh standard cage 7 days after the lesion surgery and three weeks prior to transplant surgery. They then remained in their respective post-lesion housing environment, except for the part of the day when they were fed or run on the beam and for a $48 \mathrm{~h}$ period after transplant surgery (temporary housing in individual standard cages). The enriched environment condition consisted of two identical wire mesh cages, $450 \times 100 \times 145 \mathrm{~cm}$, holding 7 or 8 rats. Each enriched cage contained a large variety of junk objects (such as plastic pipes, balls, iron chains and boxes) chosen to ensure their manipulation did not involve any behaviour too similar to the beam task; these objects were changed twice a week (as per /9/).

The final number of rats in the five groups was: Standard-Graft $(\mathrm{n}=9)$, lesion + standard housing + graft; Enriched-Graft $(n=6)$, lesion + enriched housing + graft; Standard-No Graft $(n=8)$, lesion + standard housing + no graft; Enriched-No Graft $(n=7)$, lesion + enriched housing + no graft; and a non-lesioned control group $(n=11)$, sham surgery + standard housing. Data from the five additional rats were dropped from analysis: one rat initially performed the beam task but then baulked repeatedly and four rats were found to have lesions that included both excess cortex and unintended subcortical damage.

\section{Beam-walking task}

Rats were trained to cross a narrow elevated wooden beam to gain access to $4 \times 0.1 \mathrm{~g}$ chocolate pellet food reward. The $2.5 \mathrm{~cm}$ wide beam was 200 $\mathrm{cm}$ long, with a $20 \times 20 \mathrm{~cm}$ tapered start platform and a $20 \times 20 \times 20 \mathrm{~cm}$ enclosed goal box. The run time taken to traverse the length of the beam (right to left only) was recorded by photoelectric cells. Three horizontal lines along the side of the beam were used to record the severity of any slip of the distal tip of the left hindlimb paw (contralateral to the lesion). The three lines were $5 \mathrm{~mm}, 15 \mathrm{~mm}$ and $50 \mathrm{~mm}$ below the beam surface. An observer recorded whether any foot slip occurred between 5 and $15 \mathrm{~mm}, 15$ and $50 \mathrm{~mm}$ or more than $50 \mathrm{~mm}$ below the beam surface, classified as a "minor," "moderate" or "major" foot slip, respectively; only the most severe error per slip was counted.

Training on the beam task began 6 weeks prior to lesion surgery. Rats were trained to cross the 2.5 $\mathrm{cm}$ wide beam after an initial 2 week familiarisation period on a $5 \mathrm{~cm}$ wide beam. Each rat received two training sessions per week ( 10 beam crossings per session) with half the animals trained on alternate days. Performance in terms of run time and error scores over the last four sessions prior to lesion surgery was used to rank order the rats, which were then allocated on a balanced basis to one of five groups. Performance after lesion surgery and prior to differential housing was evaluated using a single half-session ( 5 crossings) 6 days post-surgery to establish that the initial effects of the SMC lesion were also balanced across the four lesion groups; no group reassignments were made at this stage. Testing on the beam task recommenced 8 days after transplant surgery for 12 sessions (6 weeks) and was blind with respect to transplant status; the order in which the rats were tested was counterbalanced across groups.

We also filmed the rats on three occasions, as per Held et al. $/ 14 /$, first during the final training session, between the first and second post-grafting test sessions and then after the last test session. To facilitate filming, a sun gun was introduced during the initial training sessions and was used throughout all training/test sessions. A standardised analysis of the rats' fore- and hindlimb locomotor movements was performed (as per /14/), but we do not report these data; perhaps because the rats were run on a beam that was half the width of that of Held et al. (a standard procedure in unilateral lesion work) the pattern of limb movements observed was different or inconsistent even in controls to that reported previously and these measures produced no effects of lesion status, graft status or environment. 


\section{Lesion and transplant surgery}

All surgery was conducted under steriie conditions and subjects were anaesthetised with a ketamine/xylazine mixture $(80 \mathrm{mg} / \mathrm{kg}$ and 10 $\mathrm{mg} / \mathrm{kg}$ ). The functional area for the SMC, based on published physiological and behavioural observations $/ 13,15,22 /$, extends about $+4 \mathrm{~mm}$ to $-4 \mathrm{~mm}$ relative to bregma, and from $1 \mathrm{~mm}$ to $3 \mathrm{~mm}$ or 4 $\mathrm{mm}$ (depending on the AP axis) lateral to the midline (Fig. 1A). As much as possible of the SMC region of the right hemisphere was aspirated following demarcation of the intended area with a micro-scapel (Fig. 1A), followed by suturing of the scalp and a local application of an antibiotic/analgesic cream. The intended lesion area extended from $+4 \mathrm{~mm}$ to $-4 \mathrm{~mm}$ relative to bregma, $1.3 \mathrm{~mm}$ lateral to the midline along its medial extent, and from $1.3 \mathrm{~mm}$ to $3 \mathrm{~mm}$ lateral at the anterior border and from $1.3 \mathrm{~mm}$ to $4.5 \mathrm{~mm}$ lateral at the posterior border; some more lateral tissue, $+2 \mathrm{~mm}$ to $-1 \mathrm{~mm}$ AP relative to bregma, was also aspirated. Shamlesioned controls underwent no bone removal.
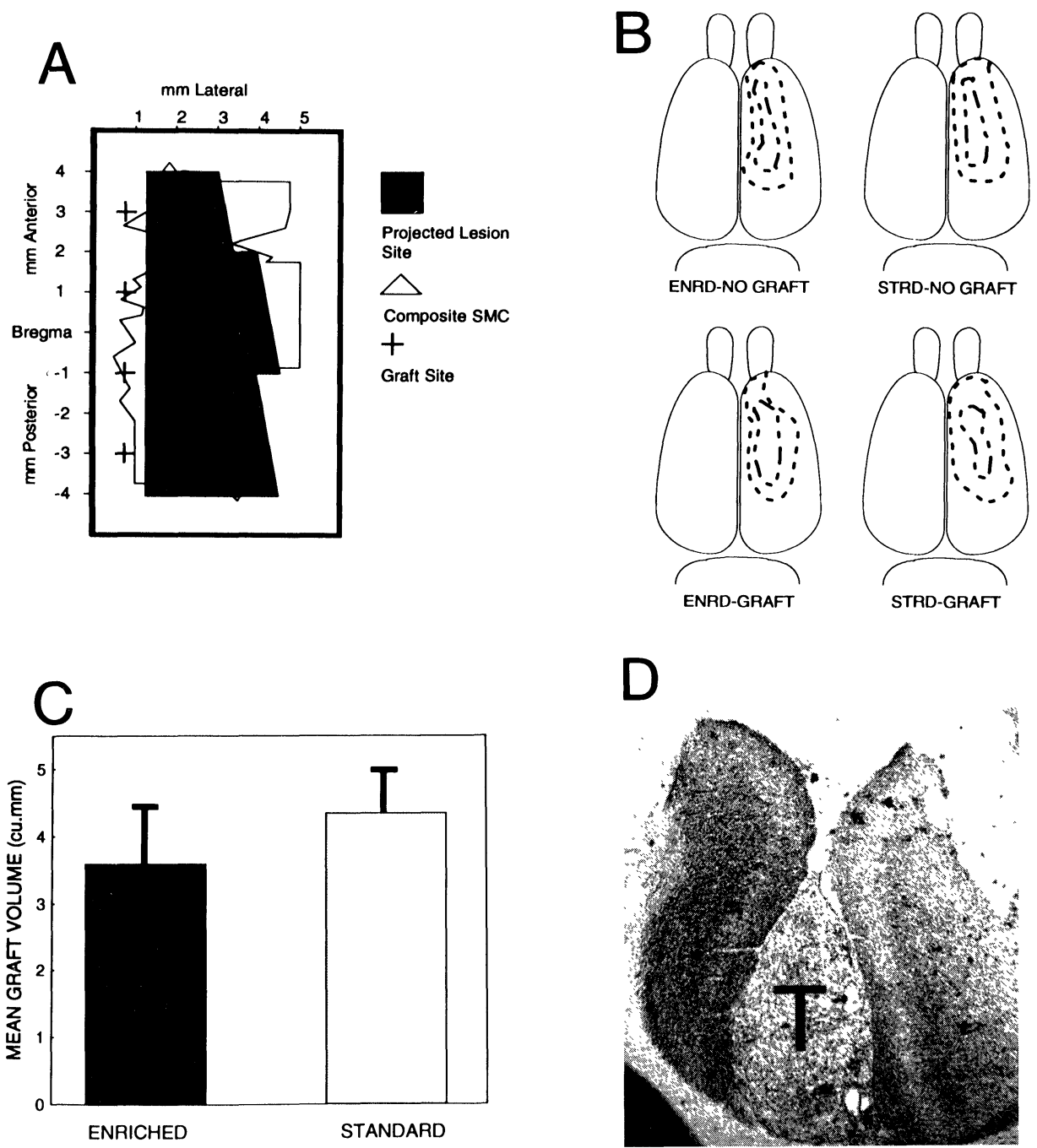

Fig. 1: A. Schematic diagram of the intended right hemisphere sensorimotor cortex lesion, relative to the composite functional area described in published work, and the adjacent graft sites for frontal suspension tissue. B. Reconstructions of the largest and smallest lesions in the four lesion groups. C. Mean graft volume in the enriched and standard-housed groups (error bars = s.e.m.). D. Photomicrograph of an acetylcholinesterase-stained section showing weaker, more irregular presence of reaction product in the graft $(\mathrm{T})$ than in the surrounding host tissue. 
Thirty days after lesion surgery, anaesthetised transplant recipients received grafts of fetal frontal cortex suspension into the dorsomedial parenchyma adjacent to the lesion. The four graft deposits were made $0.8 \mathrm{~mm}$ lateral to the midline and $2 \mathrm{~mm}$ ventral to dura, and $3 \mathrm{~mm}$ anterior, $1 \mathrm{~mm}$ anterior, 1 $\mathrm{mm}$ posterior and $3 \mathrm{~mm}$ posterior to bregma. At each site, $2 \mu$ of suspension was injected over a 2 min period with the 10- $\mu$ l Hamilton syringe left in situ for a further 3 minutes at the end of each injection. Pieces of fetal frontal cortex were obtained by removal of the olfactory bulbs and making a coronal cut just anterior to the septal region using embryonic day 20 material (E 20; crown rump length 23-24 mm) obtained from dams of the same strain that had been overdosed with nembutal. The frontal cortex tissue, free of any meninges, was rendered into a suspension using a standard procedure and one piece of fetal tissue per $10 \mu \mathrm{l}$ glucose-saline /1/. Non-transplant subjects and sham-lesioned controls were anaesthetised and their skulls exposed.

\section{Histological analysis}

At the conclusion of testing all subjects were sacrificed by nembutal overdose and perfused with saline followed by $4 \%$ formalin. The extracted brains were placed in $4 \%$ formalin at $4^{\circ} \mathrm{C}$ for 48 hours and then refrigerated in a long-term sucrose formalin solution. Frozen $50 \mu \mathrm{l}$ sections were cut on a cryostat and every $5^{\text {th }}$ and $6^{\text {th }}$ section was stained for cresyl violet and $\mathrm{AChE}$ respectively. A graphics tablet was used to trace camera lucida images of the cross-sectional area of any transplant material and then transplant volume was obtained as follows $/ 9 /$ : $\mathrm{V}=\Sigma \mathrm{A} \times \mathrm{T} \times \mathrm{F}$, where $\mathrm{V}$ is the volume in $\mathrm{mm}^{3}, \Sigma \mathrm{A}$ is the sum of the traced areas in $\mathrm{mm}^{2}$ (corrected for magnification), $T$ is the section thickness $(50 \mu)$, and $F$ is the frequency of the sections used $(1: 6)$.

\section{RESULTS}

\section{Histology}

As shown in Fig. 1B, the right hemisphere cortical lesions were comparable in the four lesion groups and produced moderate to extensive damage to the intended target region; no damage to subcortical regions was present in acceptable lesions. There was no relationship between the rank-ordered extent of cortical lesion and the behavioural measures of run time and error frequency on the post-lesion pre-grafting session ( $\mathrm{r}$ $=-0.03$ and $\mathrm{r}=+0.09$, respectively, $\mathrm{N}=30$; Spearman correlations).

Viable grafts were present in all grafted rats with acceptable lesions (only an additional enriched rat, that had an unacceptable lesion, provided no evidence of any surviving graft) but the mean total graft volume did not differ between the enriched and standard housing conditions ( $t$ value $<1.0$; Fig. 1C). The grafts were usually well-delineated, bordered by glial cells, and typically evident as small well-vascularized discrete masses. They were present in or above the corpus callosum and adjacent structures in most cases, in the cortex ipsilateral to the lesion or, as shown in Fig. 1D, in the longitudinal fissure, presumably because the left hemisphere experienced some collapse after the lesion. Some cell clumps were present in the lateral ventricles. There was an indication that larger graft tissue masses were sometimes present above the fimbria-fornix and hippocampus rather than more anteriorly. The cells in the transplants appeared normal and mature but showed no indication of laminar organisation and were densely but evenly scattered in any deposits. The graft tissues also showed no laminar organisation and less reaction product when stained for $\mathrm{AChE}$ than did the surrounding host structure (Fig. 1D). There were no significant relationships between graft size and behaviour on the beam task (for run time and error frequency, first post-grafting session, $\mathrm{df}=13, \mathrm{r}=$ $-0.37, \mathrm{p}>0.10$, and $\mathrm{r}=+0.28, \mathrm{p}>0.10$, respectively; sessions $2-12, \mathrm{df}=13, \mathrm{r}=-0.32, \mathrm{p}>$ 0.10 , and $\mathrm{r}=+0.42, \mathrm{p}>0.10$, respectively; Pearson correlations).

\section{Behavioural data}

For the sake of clarity, consistency across measures and because of problems with homogeneity of variance, data were analysed separately for the pre-lesion sessions, the first postlesion (pre-grafting) session, the first post-grafting session, and the remainder of the post-grafting sessions (2-12; for run times, error frequency and 
minor errors) or selected post-grafting sessions (3, 7 and 12; moderate errors and major errors), using ANOVAS or pair-wise Mann-Whitney $U$ tests as appropriate.

Average run time scores per session were transformed to the reciprocal; these data are shown in Fig. 2, top panel. A $2 \times 2 \times 4$ ANOVA (Graft vs No-Graft; Enriched vs Standard; repeated measure of Sessions) of the pre-lesion run times, using the non-lesioned subjects as an "isolated control" group (Statistica, Statsoft), confirmed that the five groups showed comparable beam-walking performance prior to surgery (all Fs $<1.0$ ). By contrast, run times 6 days post-lesion (MID) for the four lesion groups were considerably slower than for the nonlesioned rats (all $p$ values $<0.001$ ), but equivalent between the four lesion groups (all Fs $<1.0$ ). Hence, on this measure, as indeed for all other (foot error) scores, the initial post-lesion effects of the SMC lesions were evenly balanced across the four lesion groups.

The $2 \times 2$ ANOVA (plus isolated control group) of the run times for the first post-grafting session revealed no effects (Graft effect, $F=1.93$, df $=$ 1,36, p > 0.10; Environment effect and Graft by Environment interaction, Fs $<1.0$; Fig. 2, top panel). While the performance of all the lesion groups on this first post-grafting session had improved relative to their run times just after lesion surgery, the non-lesioned controls still had significantly faster times than any other group (vs Enriched-Graft, $p<0.05$; vs each of the other groups, $p<0.01$ ). Across post-grafting sessions 212 , the Standard-No Graft group tended to run more slowly than the other groups, but the $2 \times 2 \times$ 11 ANOVA (plus isolated control group) revealed only a Session main effect $(F=20.88, d f=10,360$, $\mathrm{p}<0.001)$ reflecting the overall improvement in running times (Graft main effect, $F=1.76$, df $=$ $1,36, p>0.10$; all others $F s<1.0$ ). The run times of the non-lesioned controls over post-grafting sessions 2-12 did not differ from those of any lesion group ( $p>0.10$ for all contrasts). To summarize the run time data, the unilateral SMC lesions produced a severe deficit in beam-walking times soon after surgery, but by one month post-lesion a milder deficit was apparent only at the beginning of retesting and the rats rapidly recovered this aspect
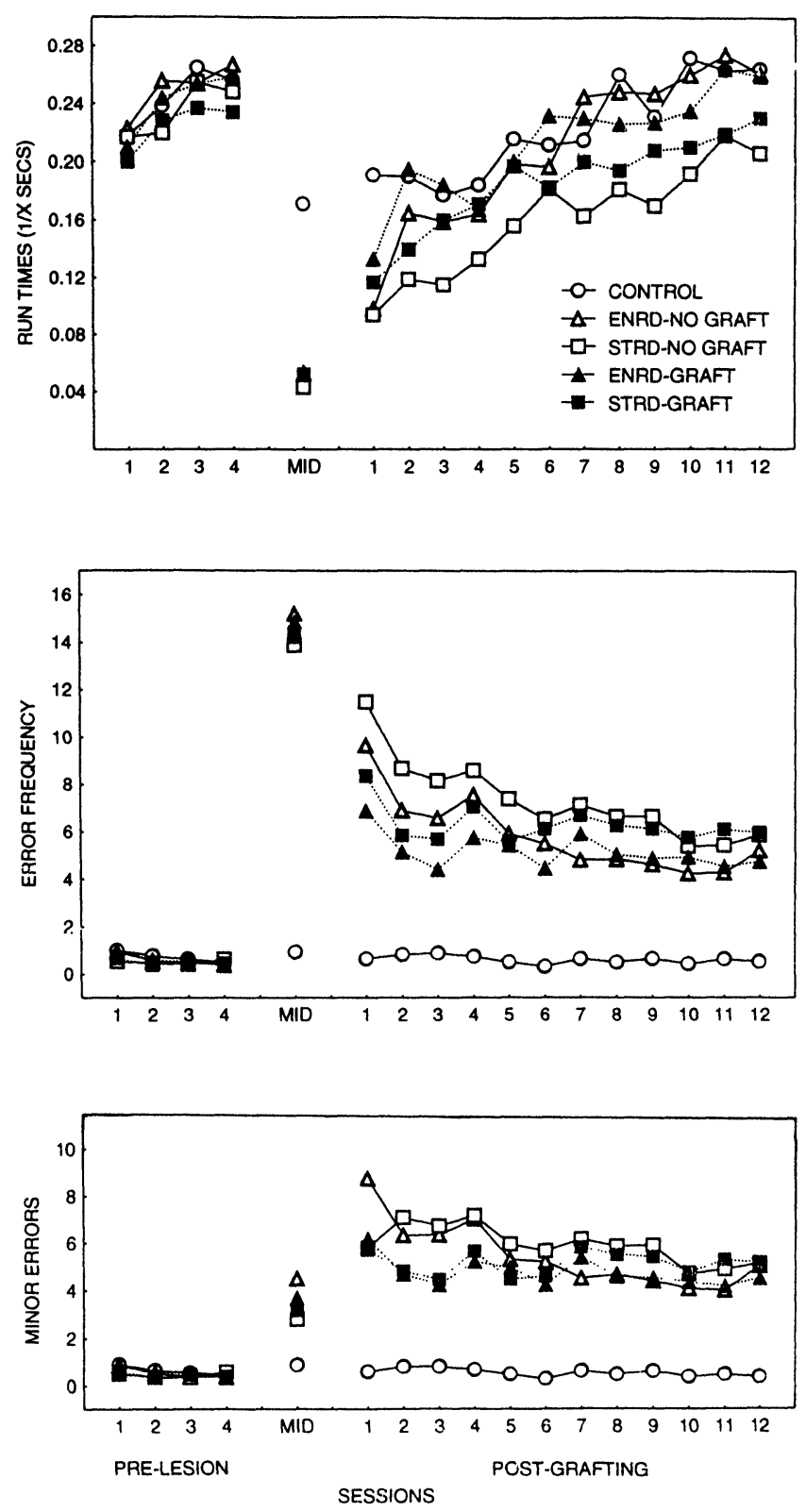

Fig. 2: Beam-walking performance of the five groups of rats for the lst four sessions prior to lesion surgery, the half-session (MID; 5 trials) prior to differential housing, and the twelve sessions post-grafting. Top panel, mean run times per trial; middle panel, mean frequency of all hindlimb foot errors per trial; bottom panel, mean number per trial of foot slips extending 5 to $15 \mathrm{~mm}$ below the edge of the beam. Control, sham-lesioned rats housed in standard group cages; Enrd-No Graft, lesion-only rats housed in an enriched environment; Strd-No Graft, lesion-only rats housed in standard group cages; Enrd-Graft, grafted rats housed in an enriched environment; Strd-Graft, grafted rats housed in standard group cages. 
of their performance, irrespective of neural grafting or environmental conditions.

The mean frequency per trial per session of total hindlimb foot-slips made by the five groups is shown in the middle panel of Fig. 2. Almost no errors were made by the end of pre-lesion training. Immediately after surgery (MID), the four lesion groups made an equal and high number of total foot slips whereas the intact rats continued to make few such errors if any (all Mann-Whitney $U$ tests for non-lesioned controls vs each lesion group, $\mathrm{p}<$ 0.001 ). There was some recovery of the number of foot-slips on the first post-grafting session relative to the prior post-lesion test, especially in grafted rats, but on this session and all subsequent sessions all lesioned groups made frequent foot slips whereas the non-lesioned rats continued to make few errors if any (Mann-Whitney $U$ comparisons between intact rats and lesion groups, all $p$ values $<0.001$ at each session). The $2 \times 2$ ANOVA (Graft vs NoGraft; Enriched vs Standard) for the first postgrafting session confirmed that the grafted rats made significantly fewer total foot-slips than the non-grafted rats $(F=5.63, \mathrm{df}=1,26, \mathrm{p}<0.05)$, but there was no Environment effect or Graft by Environment interaction $(F=1.17$ and $F<1.0$, respectively, $\mathrm{df}=1,26)$. The grafted rats' impairment on this measure improved to their mean asymptote by the second post-grafting session whereas the non-grafted rats continued to show more impairment during the early sessions. The $2 \mathrm{x}$ $2 \times 11$ ANOVA on the total foot slip frequency across the remaining 11 sessions confirmed the Graft by Session interaction $(F=4.53, \mathrm{df}=10,260$, $p<0.001)$. The non-grafted rats made more errors than grafted rats in early sessions $(p<0.05$ for simple main effects on sessions 2 and 3); only the non-grafted rats showed a Session simple main effect $(F=10.02, d f=10,260, p<0.001$, for the non-grafted rats; $F=1.38, p>0.10$, for the grafted rats). Despite the suggestion of a greater improvement by enriched rats on later sessions, there were no significant Environment effects (all Fs $<1.0$ for Environment main effect and interactions). In summary, lesion groups showed a partial recovery of their impairment of an increased number of foot-slips, this recovery was enhanced by neural grafting but not by an enriched environment, and this graft-mediated beneficial effect was apparent from the beginning of post-graft testing.

To study in more detail the nature of the lesioninduced foot-slip impairment, performance was analysed in terms of severity of foot-slip. The bottom panel of Fig. 2 shows performance in terms of "minor" foot-slips, which comprised the majority of foot errors. The high number of these foot-slips was equal across lesion groups after lesion surgery but diminished unequally across groups by the first post-grafting session. In all groups, the level of minor foot slips remained much higher throughout testing than that shown by non-lesioned rats (MannWhitney $U$ tests, all $p$ values $<0.001$ for nonlesioned group vs each lesion group at each session). A $2 \times 2$ ANOVA on minor foot slips made in the first post-grafting session for the four lesion groups showed that enriched rats made more such errors on this session $(F=4.33$, df $=1,26, p<$ $0.05)$, but the apparent Graft by Environment interaction was not significant $(\mathrm{F}=2.30, \mathrm{df}=1,26$, $p>0.10)$ nor was there a significant Graft effect ( $F$ $=2.65, \mathrm{df}=1,26, \mathrm{p}>0.10)$. ANOVA of sessions 2-12 revealed a picture that mirrored the effects found for total foot-slips, with no Environment effects (main effect and interactions, all Fs $<1.0$ ), but a highly significant Graft by Session interaction $(F=4.05, \mathrm{df}=10,260, \mathrm{p}<0.001)$ due to the grafted rats making fewer errors than non-grafted rats on early trials ( $p<0.05$ for sessions 2 and 3$)$ and only the non-grafted rats showing a reduction across trials to the levels shown by grafted counterparts (Session simple main effect for nongrafted rats, $F=7.63, \mathrm{df}=10,260, \mathrm{p}<0.001$; for grafted rats, $F=1.50, \mathrm{df}=10,260, \mathrm{p}>0.10)$.

The fewer "moderate" and "major" foot slips diminished markedly across post-grafting sessions and could not be submitted to an ANOVA because of heterogeneous and/or lack of variance. MannWhitney tests with a conservative significance level $(p<0.01)$ were therefore conducted for each measure averaged over all post-grafting sessions and at selected post-grafting sessions $(1,3,7$ and 12 ; see Fig. 3) to reduce the number of comparisons. Averaged across post-grafting sessions $1-12$, the respective mean ( \pm s.e.m) number of moderate errors and major errors per trial per lesion group was as follows: Standard-Graft, 

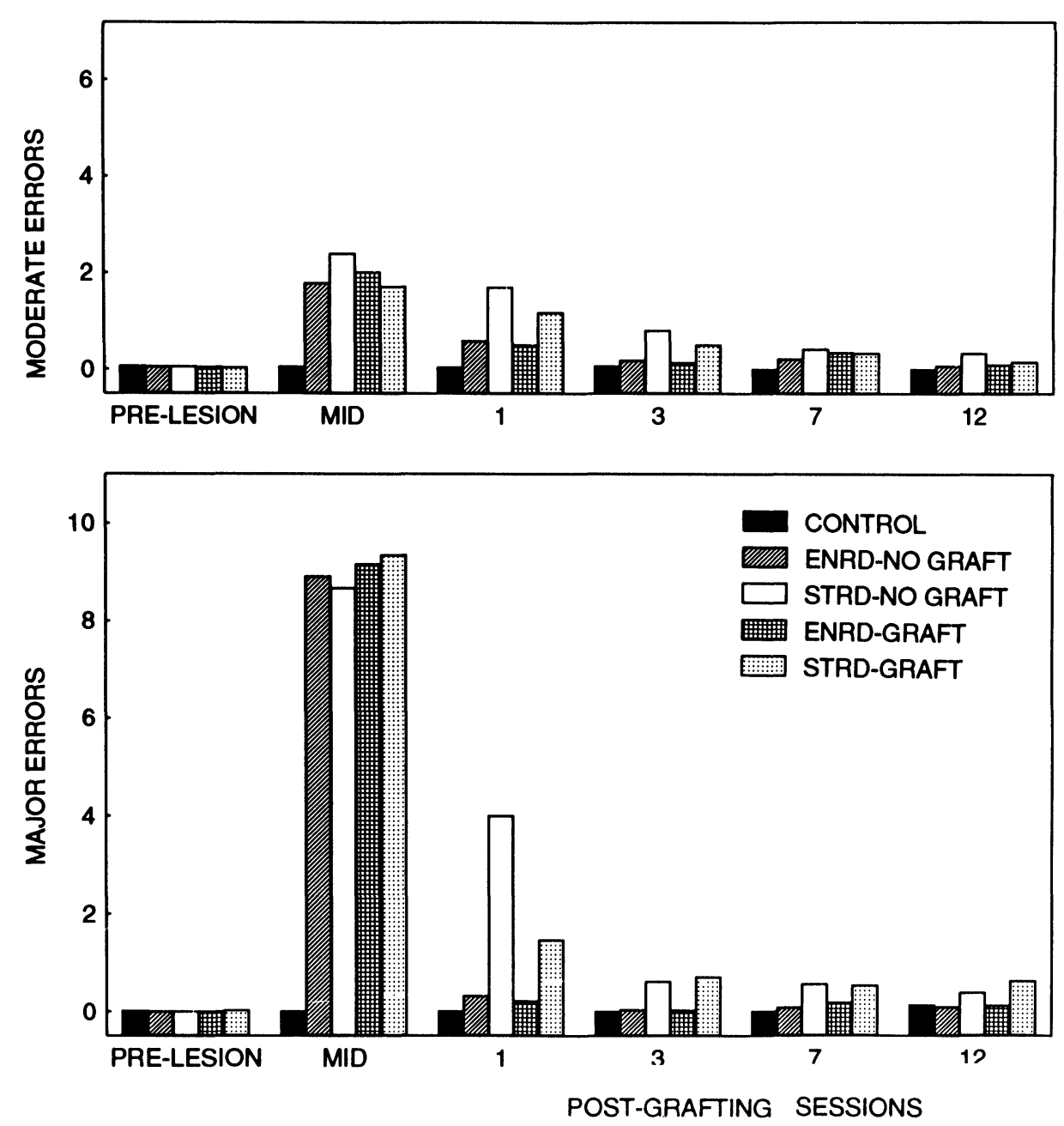

Fig. 3: Beam-walking performance of the five groups of rats in terms of hindlimb foot errors extending 15 to $50 \mathrm{~mm}$ below the edge of the beam (top panel) or greater than $50 \mathrm{~mm}$ below the beam edge (bottom panel). See Fig. 2 for legend.

$0.42 \pm 0.07$ and $0.73 \pm 0.12$; Enriched-Graft, $0.22 \pm$ 0.08 and $0.19 \pm 0.04$; Standard-No Graft, $0.56 \pm$ 0.12 and $0.85 \pm 0.23$; Enriched-No Graft, $0.21 \pm 0.04$ and $0.16 \pm 0.04$. The comparison of all grafted rats $(\mathrm{N}=15)$ versus all non-grafted rats $(\mathrm{N}=15)$ revealed no effects on either moderate or major foot slips for scores averaged across all post-grafting sessions or at any individual session (all $p$ values $>$ 0.30 ). By contrast, the comparisons of all enriched rats $(\mathrm{N}=13)$ versus all standard-housed rats $(\mathrm{N}=$ 17) confirmed that enriched rats made fewer moderate errors averaged over all post-grafting sessions $(U=40, p<0.01)$ and especially fewer major errors $(U=13, p<0.001)$. The lower number of moderate errors committed by enriched rats was significant only on the first $(U=41.5, p<$
$0.01)$ and third $(U=40.5, p<0.01)$ post-grafting sessions (for session 7 and $12, U=100$ and 69.5 respectively). The lower number of major foot errors made by enriched rats was highly significant on all sessions (all $U$ values $<26$, all $p$ values $<$ 0.001 ). Irrespective of graft status, then, enriched rats were similar to non-lesioned rats in making few moderate and major errors, whereas standard housed rats made more of these errors and continued to do so throughout testing in the case of major errors.

\section{DISCUSSION}

The present study demonstrated that fetal frontal cortex, grafted as suspension material to the intact 
tissue adjacent to a large unilateral SMC lesion in the adult rat, provided a significant early reduction of lesion-induced foot errors made performing a beam-walking task. This graft-related improvement was incomplete, however, as there was a sustained deficit in grafted rats relative to intact animals and continued beam-walking experience brought the performance of lesion-only rats to a similar level to that of the grafted rats. Nonetheless, the relative degree of behavioural recovery achieved in the present work is noteworthy by comparison with the negative data reported previously $/ 31,35 /$.

There are several differences between the current and previous work that examined the potential for fetal tissue to promote recovery of locomotor function after SMC lesions, any one or combination of which may account for their different outcomes. While our unilateral lesions were sufficient to produce clear and persistent deficits in terms of hindlimb slips off the edge of the beam, an obvious difference in the study by Slavin et al. $/ 31 /$ was that they examined recovery after bilateral SMC lesions when both run times and errors appear to be more persistently impaired. However, the significance of the severity of lesioninduced impairment is diminished by the fact that our lesions were more extensive and the resulting impairment more extreme than that in the corresponding work by Swenson et al. /35/ who found no sparing after using fetal grafts of E15 SMC tissue in neonate rats with unilateral sensorimotor lesions.

We consider that a potentially important aspect was our use of frontal tissue in suspension, placed adjacent to the lesion area and not in the lesion cavity itself. Previous behavioural work with cortical grafts has placed solid tissue directly into the lesion cavity. The success of this latter procedure in terms of graft survival has varied from excellent to poor, irrespective of behavioural outcome $/ 26,27,31,35 /$.

A particularly striking feature of the present results was the high graft survival rate obtained despite the grafts being introduced one month postlesion, which shows that such delayed use of cortical tissue can be effective to promote functional recovery, as has been the case for intrahippocampal septal suspensions $/ 7 /$. Our findings contradict the view that cortical tissue, particularly frontal tissue, may be ineffective when there is a significant delay in its placement after brain injury. Using solid frontal cortex tissue in a medial frontal cortex lesion cavity Stein et al. /34/ reported poor survival and no behavioural effects when the grafts were made 30 or more days postoperatively, unlike the survival and beneficial effects found when grafts were made up to 14 days after cortical injury. This contrast in findings may be related to differences in terms of lesion site and behaviour, but it is possible that the use of suspension material may also be beneficial in the case of medial frontal damage and delays greater than 14 days.

Previous work has highlighted the potential benefits of using frontal cortex grafts at ectopic sites, both after occipital cortex lesions and when used in combination with ganglioside treatment after SMC lesions $/ 20,31$. We have not compared the relative benefit of frontal cortex as opposed to presumptive SMC but consider that the use of a precise frontal region may be an important factor in our results, as may be the relatively late fetal age (E20) of this cortical tissue and the commencement of testing shortly after grafting. Dunnett et al. /8/ found that younger (E16) frontal grafts may exacerbate frontal lesion-induced impairments, in contrast to the early beneficial effects of older (E21) tissue, and concluded that the functional benefits of these grafts depend on the precise combination of conditions rather than reflecting a general phenomenon. The exact graft tissue used in related SMC-lesion studies is unclear, and it is apparent that no other study has used the restricted frontal region we used, but only "frontal" tissue has achieved any success previously, including related work in which frontal grafts promoted recovery of paw-reaching behaviour after anterior SMC lesions /26/.

The use of a one month lesion-graft delay reduces the likelihood of any major contribution by host trophic factors to the differential performance of grafted and non-grafted rats, although some influence by trophic elements arising from nonspecific tissue damage as part of the grafting procedure cannot be discounted. It is likely, however, that the release and/or diffusion of trophic substances, primarily from the frontal grafts, contributed to the observed restoration of 
behavioural functions. Similar speculations of the effects of frontal grafts on behavioural recovery have been made in the context of medial frontal lesions $/ 8,19,20 /$; there is in fact explicit evidence from work with frontal cortex lesions that both solid frontal grafts and cortical cell suspensions have trophic effects on the thalamus /12,29/. Given these considerations, and our delayed use of fetal frontal cortex in suspension and a brief graft-test delay, we conclude it likely that general metabolic factors (e.g., trophic factors; restitution of function in adjacent regions) rather than any specific new neuronal connections contributed to the behavioural recovery we observed. Additional work is needed to address these issues.

Postoperative enrichment produced a more subtle influence on beam-walking performance than did neural grafts. Irrespective of graft status, enriched rats made few if any moderate and major foot errors, unlike their standard-housed counterparts, but the overall number of errors was not significantly reduced. The number of moderate and major errors committed was small by comparison to minor errors, and enrichment may have prevented the occurrence of any severe errors by an immediate compensation of foot faults that may otherwise have been moderate or major errors. In fact enriched rats actually made more minor errors but fewer of the more severe errors at the beginning of post-graft testing.

Our unilateral lesions produced only a transient deficit on run times but overall foot-slip errors were persistently increased irrespective of housing conditions. Held et al. /14/ reported that both preand postoperatively enriched adult rats with large bilateral SMC lesions showed reduced deficits on beam-running times compared with rats housed in postoperative "impoverished" conditions. Held et al. /14/ did not report foot errors but found that movement patterns were normalised only by preoperative enrichment, so it is possible that their postoperatively enriched rats ran quickly but still made some minor errors. There is also some limited evidence that postoperatively enriched rats are less likely than individually-housed rats to show any rear foot faults (unspecified) after both bilateral and unilateral anterior frontal cortex lesions; unfortunately, these animals were examined only on the last three training trials when, unlike other rats, the individually-housed rats with lesions made only one or two errors per trial /18/. The apparent variance between these studies' findings and our results may be due to several factors, including our use of large unilateral SMC lesions as opposed to either medial frontal lesions or bilateral SMC lesions, our use of explicit and sensitive measures of foot faults throughout post-graft testing and our explicit avoidance of any "beam-like" objects in the enriched environments to minimise simple transfer of training effects.

Surprisingly little work has addressed the potential interactive effects of postoperative enrichment and neural grafts $/ 36 /$. Part of the rationale of the present study was to explore the suggestion of Stein et al. /34/ to study the effects of post-lesion enrichment imposed early after CNS injury combined with neural transplantation imposed at a later time. The lack of any interactive effect of enrichment and neural grafting in the current study contrasts with a previous study in which only rats with fimbria-fornix lesions that received a combination of septal grafts at lesion surgery and enrichment immediately thereafter, but not those that received either alone, showed some sparing of maze learning ability $/ 16 /$. The specific influence of enrichment in the present study was to minimise the occurrence of severe errors made on the beam, whereas the grafts but not enrichment produced a significant reduction of foot faults in general. In the present conditions, then, enrichment was unable to augment the general degree of recovery beyond that produced by a neural transplant.

It is possible that better recovery of function after sensorimotor cortex lesions might occur with enrichment combined with a shorter lesion-graft delay than was used in the present work, perhaps through an early enhancement of potential grafthost connections with a short lesion-graft delay, or when more time is allowed for a graft/environment interaction to take place. Alternatively, improved recovery and transplant-host integration may be evident with cell suspensions placed in the sensorimotor cortex area denervated by an excitotoxic rather than aspirative lesion. Nonetheless, the present study shows that suspension grafts of fetal cortex survive transplantation at four weeks after lesion surgery and promote functional recovery on a beam-walking task. In summary, suspension grafts 
of late fetal frontal cortex provided incomplete functional recovery of beam-walking errors induced by unilateral SMC lesions in adult rats, but this effect was not enhanced by a postoperatively enriched environment.

\section{ACKNOWLEDGEMENTS}

We are grateful to The Neurological Foundation of New Zealand Inc. and the University of Canterbury for financial assistance. We also thank Mrs. P. Meatchem, Mr. D. Covich, Mr. G. Lewis and Mr. R. Phillips for technical assistance.

\section{REFERENCES}

1. Björklund A, Stenevi U, Schmidt RH, Dunnett SB, Gage FH. Intracerebral grafting of neuronal cell suspensions. II. Survival and growth of nigral cell suspensions implanted in different brain sites. Acta Physiol Scand Suppl 1983; 522: 9-16.

2. Castro AJ, Tønder N, Sunde N, Zimmer J. Fetal cortical transplants in the cerebral hemisphere of newborn rats: a retrograde fluorescent analysis of connections. Exp Brain Res 1987; 66: 533-542.

3. Castro AJ, Tønder N, Sunde N, Zimmer J. Fetal cortical transplants grafted into the cerebral cortex of newborn rats. Analysis of efferents from the basal forebrain, locus coeruleus and midline raphe. Exp Brain Res 1988; 69: 613-622.

4. Castro AJ, Zimmer J, Sunde NA, Bold EL. Transplantation of fetal cortex to the brain of newborn rats. A retrograde fluorescent analysis of callosal and thalamic projections from transplant to host. Neurosci Lett 1985; 60: 283-288.

5. Dalrymple-Alford JC, Kelche CR. Behavioral effects of preoperative and postoperative differential housing in rats with brain lesions: A review. In: Will B, Schmitt P, Dalrymple-Alford JC, eds, Brain Plasticity, Learning and Memory: Advances in Behavioral Biology, 28, Plenum Press, New York, 1985: 441-458.

6. Dunnett SB. Neural transplantation in animal models of dementia. Eur J Neurosci 1990; 2: 567-587.

7. Dunnett SB, Martel FL, Rogers DC, Finger S. Factors affecting graft amelioration of differential reinforcement of low rates (DRL) and activity deficits after fimbria-formix lesions. Rest Neurol Neurosci 1989; 1: 93-92.

8. Dunnett SB, Ryan CN, Levin PD, Reynolds M, Bunch ST. Functional consequences of embryonic neocortex transplanted to rats with prefrontal cortex lesions Behav Neurosci 1987; 101: 489-503.

9. Dunnett SB, Whishaw IQ, Bunch ST, Fine A
Acetylcholine-rich neuronal grafts in the forebrain of rats: Effects of environmental enrichment, neonatal noradrenaline depletion, host transplantation site and regional source of embryonic donor cells on graft size and acetylcholinesterase-positive fibre outgrowth. Brain Res 1986; 378: 357-373.

10. Gonzalez MF, Sharp FR. Fetal frontal cortex transplanted to injured motor/sensory cortex of adult rats. I. NADPH diaphorase neurons and their connections. J Neurosci 1987; 7: 2991-3001.

11. Gonzalez MF, Sharp FR, Loken JE. Fetal frontal cortex transplanted to injured motor/sensory cortex of adult rats: Reciprocal connections with host thalamus demonstrated with WGA-HRP. Exp Neurol 1988; 99: 154-165.

12. Haun F, Cunningham TJ. Cortical transplants reveal CNS trophic interactions in situ. Dev Brain Res 1984; 15: 290-294.

13. Hall RD, Lindholm EP. Organisation of motor and somatosensory neocortex in the albino rat. Brain Res 1974; 66: 23-38.

14. Held JM, Gordon J, Gentile AM. Environmental influences on locomotor recovery following cortical lesions in rats. Behav Neurosci 1985; 4: 678-690.

15. Hicks SP, D'Amato CJ. Motor-sensory cortexcorticospinal system and developing locomotion and placing in rats. Am J Anat 1975; 143: 1-41.

16. Kelche C, Dalrymple-Alford JC, Will B. Housing conditions modulate the effects of intracerebral grafts in rats with brain lesions. Behav Brain Res 1988; 28: 287-295.

17. Kimble DP. Functional effects of neural grafting in the mammalian central nervous system. Psych Bull 1990; 108: $462-479$.

18. Kolb B, Gibb R. Environmental enrichment and cortical injury: Behavioral and anatomical consequences of frontal cortex lesions. Cerebral Cortex 1991; 1: 189-198.

19. Kolb B, Reynolds B, Fantie B. Frontal cortex grafts have opposite effects at different postoperative recovery times. Behav Neural Biol 1988; 50: 193-206.

20. Lescaudron L, Stein DG. Functional recovery following transplants of embryonic brain tissue in rats with lesions of visual, frontal and motor cortex: Problems and prospects for future research. Neuropsychologia 1990; 28: 585-599.

21. Levin BE, Dunn-Meynell A, Sved AF. Functional integration of fetal cortical grafts into the afferent pathway of the rat somatosensory cortex (SmI). Brain Res Bull 1987; 19: 723-734.

22. Neafsey EJ, Bold EL, Haas G, Hurley-Geis KM, Quirk $\mathrm{G}$, Sievert CF, Terreberry RR. The organization of the rat motor cortex: A microstimulation study. Brain Res Rev 1986; 11: 77-96.

23. Neafsey EJ, Sørensen JC, Tønder NB, Castro AJ. Fetal cortical transplants into neonatal rats respond to 
thalamic and peripheral stimulation in the adult. An electrophysiological study of single-unit activity. Brain Res 1989; 493: 33-40.

24. Nieto-Sampedro $M$, Lewis ER, Cotman CW, Manthorpe M, Skaper SD, Barbin GR, Longo FM, Varon S. Brain-injury causes a time-dependent increase in neuronotrophic activity in the lesion site. Science 1982; 217 : 860-861.

25. Nieto-Sampedro M, Whittemore SR, Needels DL, Larson JL, Cotman $\mathrm{CW}$. The survival of brain transplants is enhanced by extracts from injured brain. Proc Natl Acad Sci 1984; 81: 6250-6254.

26. Plumet J, Cadusseau J, Roger M. Skilled forelimb use in the rat: Amelioration of functional deficits resulting from neonatal damage to the frontal cortex by neonatal transplantation of fetal cortical tissue. Rest Neurol Neurosci 1991; 3: 135-147.

27. Sandor R, Gonzalez MF, Moseley M, Sharp FR. Motor deficits are produced by removing some cortical transplants grafted into injured sensorimotor cortex of neonatal rats. J Neur Transplant Plast 1991; 2: 221233.

28. Senatorov VV, Obuhova GP, Fülöp $Z$. Electrophysiological and morphological properties of embryonic neocortical grafts developing in different regions of the host rat brain. J Neur Transplant Plast 1991; 2: 125-140.

29. Sharp FR, Gonzalez MF. Fetal cortical transplants ameliorate thalamic atrophy ipsilateral to neonatal frontal cortex lesions. Neurosci Lett 1986; 71: 247251.

30. Sharp FR, Gonzalez MF, Sagar SM. Fetal frontal cortex transplanted to injured motor/sensory cortex of adult rats. II. VIP, somatostatin, and NPY immunoreactive neurons. J Neurosci 1987; 7: 30023015.

31. Slavin M, Held JM, Basso MD, Lesensky S, Curran E, Gentile AM, Stein DG. Fetal brain tissue transplants and recovery of locomotion following damage to sensorimotor cortex in rats. In: Gash DM, Sladek JR, eds, Progress in Brain Research. Amsterdam: Elsevier Science Publishers, 1988: 33-38

32. Soares H, McIntosh TK. Fetal cortical transplants in adult rats subjected to experimental brain injury. $\mathrm{J}$ Neur Transplant Plast 1991; 2: 207-220.

33. Stein DG, Mufson EJ. Morphological and behavioral characteristics of embryonic brain tissue transplants in adult, brain-damaged subjects. In: Azmitia EC, Björklund A, eds, Annals of the NY Academy of Sciences: Cell and Tissue Transplantation into the Adult Brain 1987; 495: 444-471.

34. Stein DG, Palatucci C, Kahn D, Labbe R. Temporal factors influence recovery of function after embryonic brain tissue transplants in adult rats with frontal cortex lesions. Behav Neurosci 1988; 102: 260-267.

35. Swenson RS, Danielsen EH, Klausen BS, Erlich E, Zimmer J, Castro AJ. Deficits in beam-walking after neonatal motor cortical lesions are not spared by fetal cortical transplants in rats. J Neur Transplant Plast 1989; 1: 129-133.

36. Will B, Kelche C. Environmental approaches to recovery of function from brain damage: A review of animal studies (1981 to 1991). In: Rose FD, Johnson DA, eds, Recovery of Function following Brain Damage. New York: Plenum Press, 1992. 

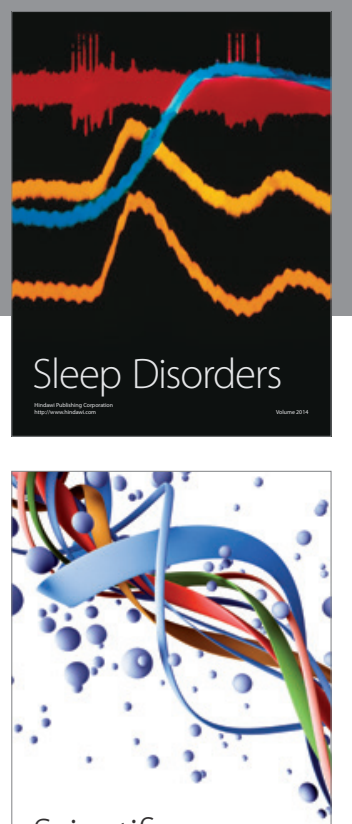

Scientifica
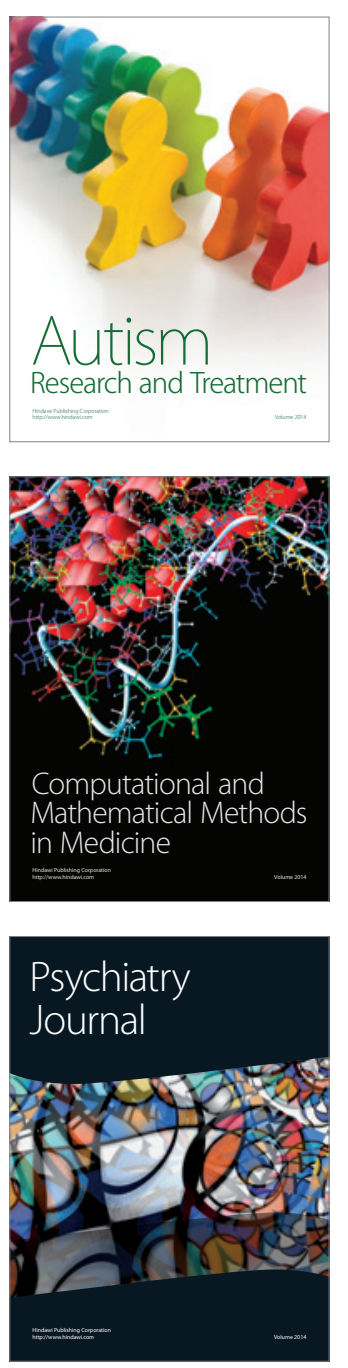
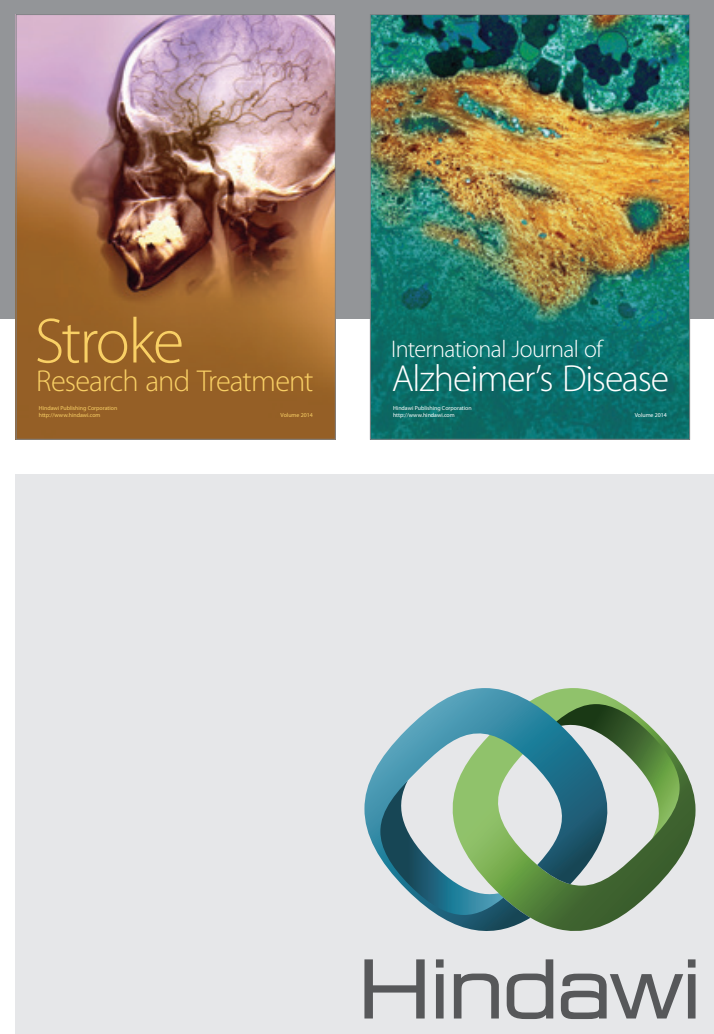

Submit your manuscripts at

http://www.hindawi.com
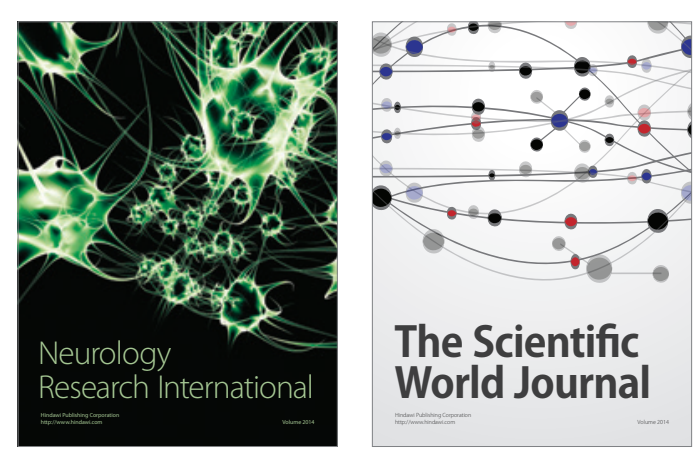

The Scientific World Journal

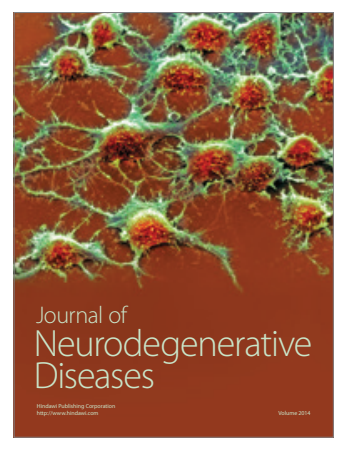

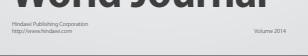

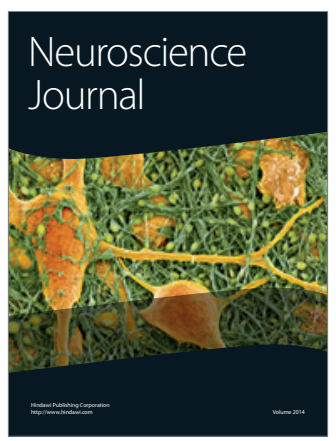

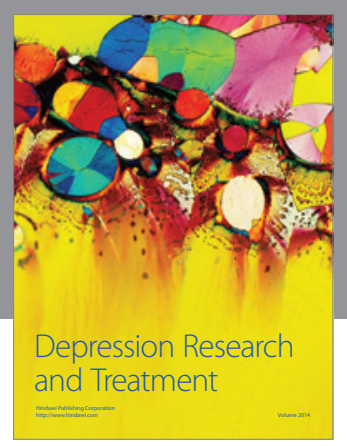
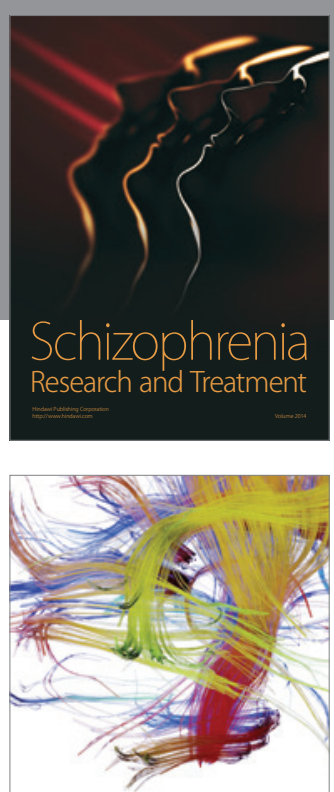

Brain Science

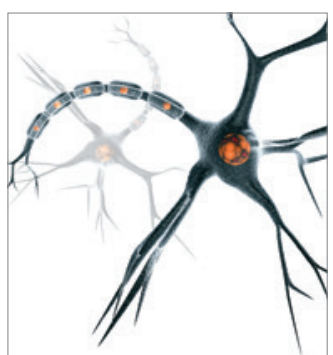

Neural Plasticity
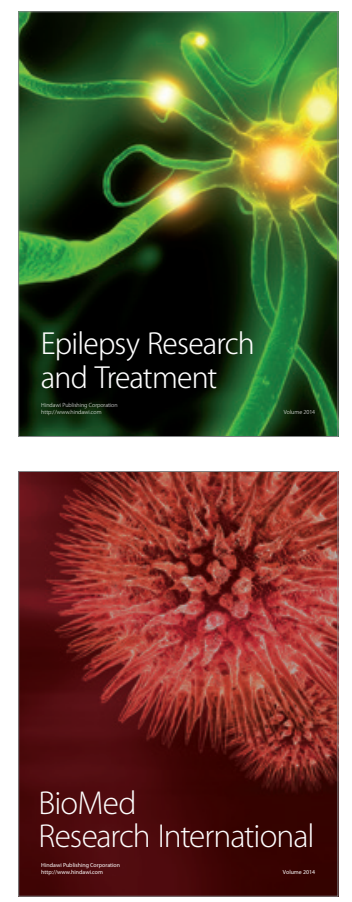

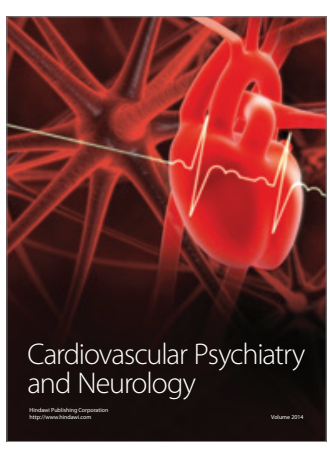

Parkinson's

Disease
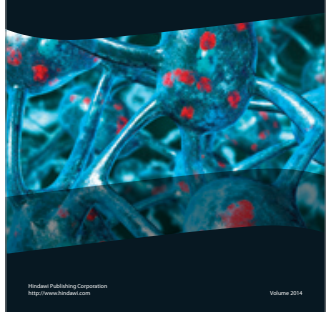